\title{
A simple model for 2D image upconversion of incoherent light
}

\section{Dam, Jeppe Seidelin; Pedersen, Christian; Tidemand-Lichtenberg, Peter}

\section{Published in:}

Proceedings of SPIE - The International Society for Optical Engineering

Link to article, DOI:

$10.1117 / 12.873820$

Publication date:

2011

Document Version

Publisher's PDF, also known as Version of record

Link back to DTU Orbit

Citation (APA):

Dam, J. S., Pedersen, C., \& Tidemand-Lichtenberg, P. (2011). A simple model for 2D image upconversion of incoherent light. Proceedings of SPIE - The International Society for Optical Engineering, 7917, 791714. https://doi.org/10.1117/12.873820

\section{General rights}

Copyright and moral rights for the publications made accessible in the public portal are retained by the authors and/or other copyright owners and it is a condition of accessing publications that users recognise and abide by the legal requirements associated with these rights.

- Users may download and print one copy of any publication from the public portal for the purpose of private study or research.

- You may not further distribute the material or use it for any profit-making activity or commercial gain

- You may freely distribute the URL identifying the publication in the public portal 


\title{
A simple model for 2-D upconversion of incoherent light
}

\author{
Jeppe Seidelin Dam, Christian Pedersen, and Peter Tidemand-Lichtenberg \\ DTU Fotonik, Frederiksborgvej 399, 4000 Roskilde, Denmark
}

\begin{abstract}
We present a simple theoretical model for 2 dimensional (2-D) image up-conversion of incoherent light. While image upconversion has been known for more than 40 years, the technology has been hindered by very low conversion quantum efficiency $\left(\sim 10^{-7}\right)$. We show that our implementation compared to previous work can result in a feasible system: Using intracavity upconversion and Quasi Phase Matching (QPM) nonlinear materials provide increased conversion efficiency. Using a QPM crystal and choosing the wavelengths so the first order term in the phasematch wavelength acceptance vanishes, results in very large wavelength acceptance. This work describes how the bandwidth acceptance can be predicted and designed. This gives promise of a new way to make infrared imaging devices with tunable spectral sensitivity.
\end{abstract}

Keywords: Upconversion, thermal imaging, infrared, Sum Frequency Generation

\section{INTRODUCTION}

Image upconversion has been considered as a possible means to obtain an infrared imaging device with particular emphasis from 1965 to $\sim 1980$ where the research field was practically abandoned due to low conversion efficiencies [1][4]. At that time, the best demonstrated continuous wave quantum efficiencies were on the order of $10^{-7}$. Of course, such low quantum efficiencies are prohibitive of any real use of upconversion imaging. Also the demonstrated image resolutions were limited to approximately 20x20 image elements. In recent work, the present authors have shown 6 orders of magnitude higher conversion efficiencies [5], and more than 3 orders of magnitude higher resolution [6], thus making the idea of having an efficient device based on these principles much less unthinkable. In this work, we will discuss how to optimize another important parameter for upconversion, namely the bandwidth acceptance. Quasi phasematching is a well known technique to make any wavelengths phasematch even along the crystal axes with highest nonlinearity. In this work, we will derive a way to predict large acceptance bandwidths. Optimizing this parameter is interesting since twice the bandwidth will usually result in twice the amount of upconverted photons when imaging a wide spectral source. Also, if narrow bandwidth upconversion should be desired for whatever reason, the principles presented here, can be used to optimize for that as well.

\section{THEORY}

In sum frequency generation, two longer, but not identical, wavelength photons combine and create a photon with shorter wavelength. Thanks to energy conservation the wavelengths are related like shown in Eq. (1).

$$
\frac{1}{\lambda_{3}}=\frac{1}{\lambda_{1}}+\frac{1}{\lambda_{2}} \Rightarrow \lambda_{3}=\frac{\lambda_{1} \lambda_{2}}{\lambda_{1}+\lambda_{2}}
$$

Here, $\lambda_{1}$, and $\lambda_{2}$ are the wavelengths that are added together to produce $\lambda_{3}$. In the upcovnersion experiment $\lambda_{1}$ is the wavelength that is upconverted, and $\lambda_{2}$ is the upconversion laser wavelength.

The individual wavelengths in the upconverter will have different wavenumbers $k_{i}$ and refractive indices $n\left(\lambda_{i}\right)$, $i \in\{1,2,3\}$ as detailed in Eq. (2).

Nonlinear Frequency Generation and Conversion: Materials, Devices, and Applications X, edited by Konstantin L. Vodopyanov, Proc. of SPIE Vol. 7917, 791714 · @ 2011 SPIE

CCC code: $0277-786 \mathrm{X} / 11 / \$ 18 \cdot$ doi: $10.1117 / 12.873820$ 


$$
k_{i}=\frac{2 \pi n\left(\lambda_{i}\right)}{\lambda_{i}}
$$

For upconversion of monochromatic light, the poling period of the upconverting crystal can be designed to quasi phasematch, Eq. (3).

$$
\Delta k=k_{3}-k_{2}-k_{1} \pm K_{Q P M}=0
$$

Where $K_{Q P M}=\frac{\pi}{\Lambda}$, and $\Lambda$ is the domain width in the period poling (i.e. half the period). Thus, by properly designing the poling period, quasi phase matching is achieved. However, for upconversion of light from a broad spectrum light source, there will be imperfect phase-match for some wavelengths. When $\Delta k \neq 0$, the conversion efficiency is reduced by a factor $\operatorname{sinc}^{2}\left(\frac{\Delta k L}{2}\right)$ where $L$ is the length of the nonlinear crystal. To examine the bandwidth acceptance of the upconverter, we note that the powerful intracavity laser used for upconversion is monochromatic $\left(\lambda_{2}\right)$. By taking the derivative of Eq. (3) with respect to $\lambda_{1}$ we get Eq. (4).

$$
\frac{\partial \Delta k}{\partial \lambda_{1}}=-\frac{2 \pi}{\lambda_{1}}\left(\frac{n_{3}-n_{1}}{\lambda_{1}}+\frac{\partial n_{1}}{\partial \lambda_{1}}-\frac{\lambda_{2}}{\lambda_{1}+\lambda_{2}} \frac{\partial n_{3}}{\partial \lambda_{3}}\right)
$$

The full width half $\max$ (FWHM) wavelength acceptance is derived in Eq. (5), which is valid for small bandwidths.

$$
\Delta \lambda_{1, F W H M}=2 \cdot \frac{2 \pi 0.4429}{L} \cdot\left|\frac{\partial \Delta k}{\partial \lambda_{1}}\right|^{-1}=\frac{0.8858 \cdot \lambda_{1}}{L} \cdot\left|\frac{n_{3}-n_{1}}{\lambda_{1}}+\frac{\partial n_{1}}{\partial \lambda_{1}}-\frac{\lambda_{2}}{\lambda_{1}+\lambda_{2}} \frac{\partial n_{3}}{\partial \lambda_{3}}\right|^{-1}
$$

Broadband upconversion will happen when the last term in Eq. (5) approaches zero. In those cases either a second order approximation or a full numerical analysis is then needed to calculate the bandwidth of the upconverter.

For mixing light near $766 \mathrm{~nm}\left(\lambda_{1}\right)$ with a $1342 \mathrm{~nm}$ laser $\left(\lambda_{2}\right)$ in a $10 \mathrm{~mm}$ properly designed pp:KTP crystal, we obtain a FWHM acceptance bandwidth of $0.25 \mathrm{~nm}$, when using the sellmeier equations from Kato et al [7].

For upconversion of a broadband source, the bandwidth of the upconverted wavelength is related to the bandwidth of the incoming radiation as follows:

$$
\Delta \lambda_{3, F W H M} \approx \Delta \lambda_{1, F W H M} \frac{\partial \lambda_{3}}{\partial \lambda_{1}}=\Delta \lambda_{1, F W H M}\left(\frac{\lambda_{3}}{\lambda_{1}}\right)^{2}
$$

Inserting numbers in Eq. (6) gives the bandwidth of upconverted light at $488 \mathrm{~nm}\left(\lambda_{3}\right)$ to be $0.10 \mathrm{~nm}$. If the bandwidth is measured in frequency, it is identical at $\lambda_{1}$ and $\lambda_{3}$ due to energy conservation, assuming single frequency $\lambda_{2}$.

Furthermore, Eq. (6) indicates that if broadband upconversion is performed, then any spectral information present in the incident radiation will also be present in the upconverted spectrum, only at a compressed spectral bandwidth. 
In order to predict where broadband upconversion is possible a different approach to this analysis is required. First, note that since $\lambda_{2}$ is constant, the frequency-shift of $\lambda_{3}$ will be exactly the same as for $\lambda_{1}$. By taking the derivative of Eq. (3) with respect to $v_{1}$ we get Eq. (7).

$$
\frac{\partial \Delta k}{\partial v_{1}}=\frac{\partial k_{3}}{\partial v_{1}}-\frac{\partial k_{1}}{\partial v_{1}}=\frac{\partial k_{3}}{\partial v_{3}}-\frac{\partial k_{1}}{\partial v_{1}}
$$

Thus, if we want to obtain broadband upconversion we should make sure that $\frac{\partial \Delta k}{\partial v_{1}}=0$ which means that $\Delta k$ to first order is constant under changes of $v_{1}$. From Eq. (7) this is identical to the requirement in Eq. (8).

$$
\frac{\partial k_{3}}{\partial v_{3}}=\frac{\partial k_{1}}{\partial v_{1}}
$$

\section{EXAMPLE CALCULATIONS}

We can now use this theory to derive how to obtain broad spectral acceptance of a particular wavelength in a particular crystal. If broad acceptance bandwidth is desired $\lambda_{1}$ and $\lambda_{3}$ should be chosen to have identical $\frac{\partial k}{\partial v}$. From this selection of $\lambda_{1}$ and $\lambda_{3}, \lambda_{2}$ is given by energy conservation (Eq. (1)). To obtain phasematch for these wavelength a proper $\mathrm{K}_{\mathrm{QPM}}$ (i.e. required poling period to obtain quasi phase matching) can be calculated from Eq. (3). Now if we plot $\frac{\partial k}{\partial v}$ as calculated from Sellmeier equations for KTP, we obtain Fig. 1. 


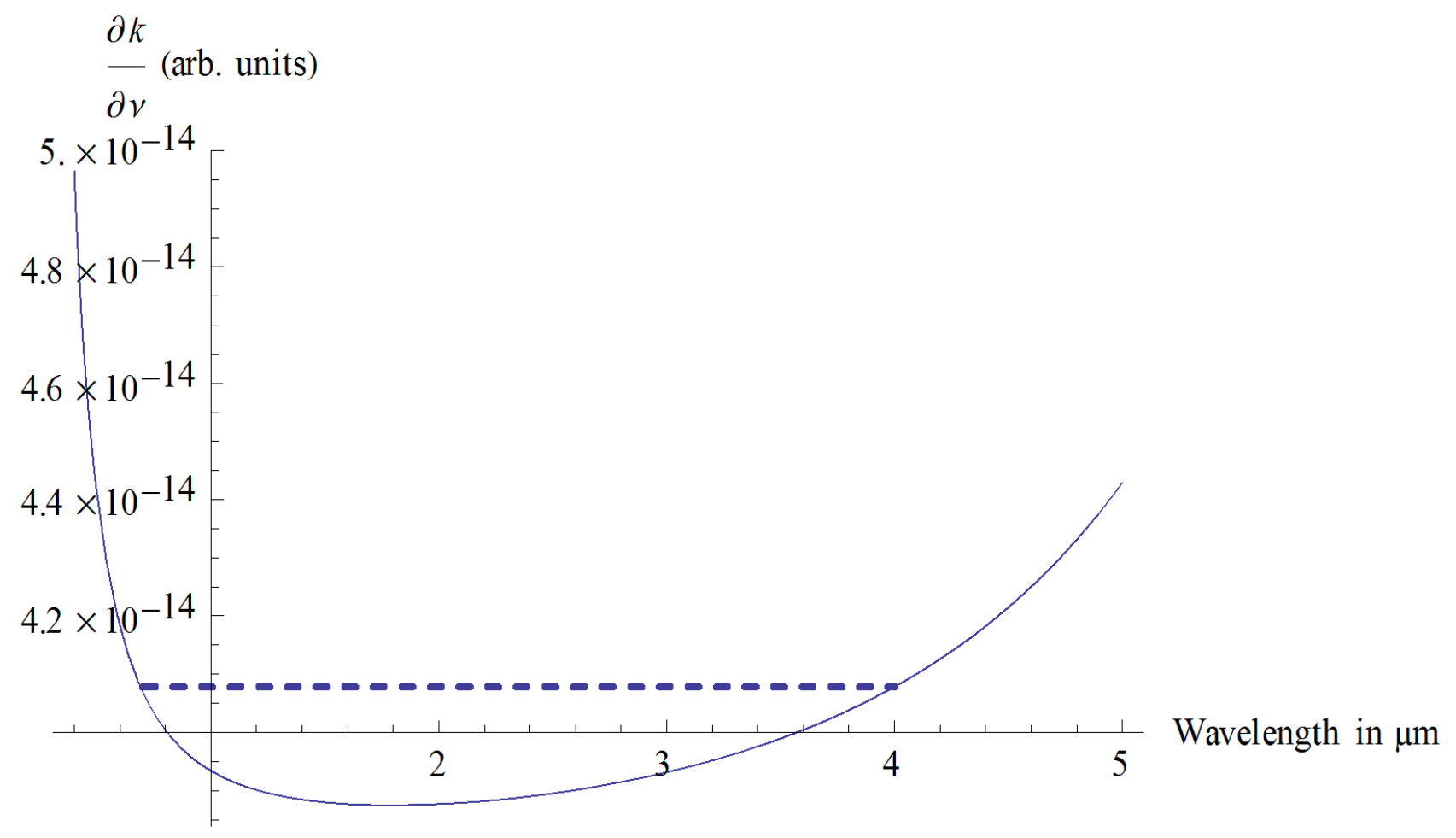

Figure 1. $\frac{\partial k}{\partial v}$ as calculated from Sellmeier equatuions, plotted against wavelength. Broad acceptance bandwidth can be gained by choosing $\lambda_{I}$ and $\lambda_{3}$ to have equal $\frac{\partial k}{\partial v}$. This can e.g. be done by choosing $\lambda_{1}=4 \mu \mathrm{m}$ and $\lambda_{3}=0.694 \mu \mathrm{m}$ as indicated by the dashed line. This would require an upconversion laser wavelength $\left(\lambda_{2}\right)$ of $0.835 \mu \mathrm{m}$.

From the example presented in Fig. 4 , it is easily calculated that to phase match upconversion of $4 \mu \mathrm{m}$ and $0.835 \mu \mathrm{m}$ to $0.694 \mu \mathrm{m}$, requires a poling period of $21.4 \mu \mathrm{m}$. A full numerical analysis reveals that in this situation the FWHM bandwidth will be $\sim 225 \mathrm{~nm}$ for a $10 \mathrm{~mm}$ long crystal.

If, for some reason, a narrow acceptance bandwidth is desired, there should be chosen wavelengths that have vastly different $\frac{\partial k}{\partial v}$ values. This can be done by mixing $4 \mu \mathrm{m}$ light with $532 \mathrm{~nm}$ light to generate $470 \mathrm{~nm}$ light. In this case, a poling period of $10.85 \mu \mathrm{m}$ is required. The bandwidth of upconversion for a $10 \mathrm{~mm}$ long crystal will be $6.7 \mathrm{~nm}$. Thus, by properly choosing the mixing wavelengths, either quite narrow or comparatively wide acceptance bandwidths can be obtained. The bandwidth acceptance for these cases is plotted in Fig. 2. 


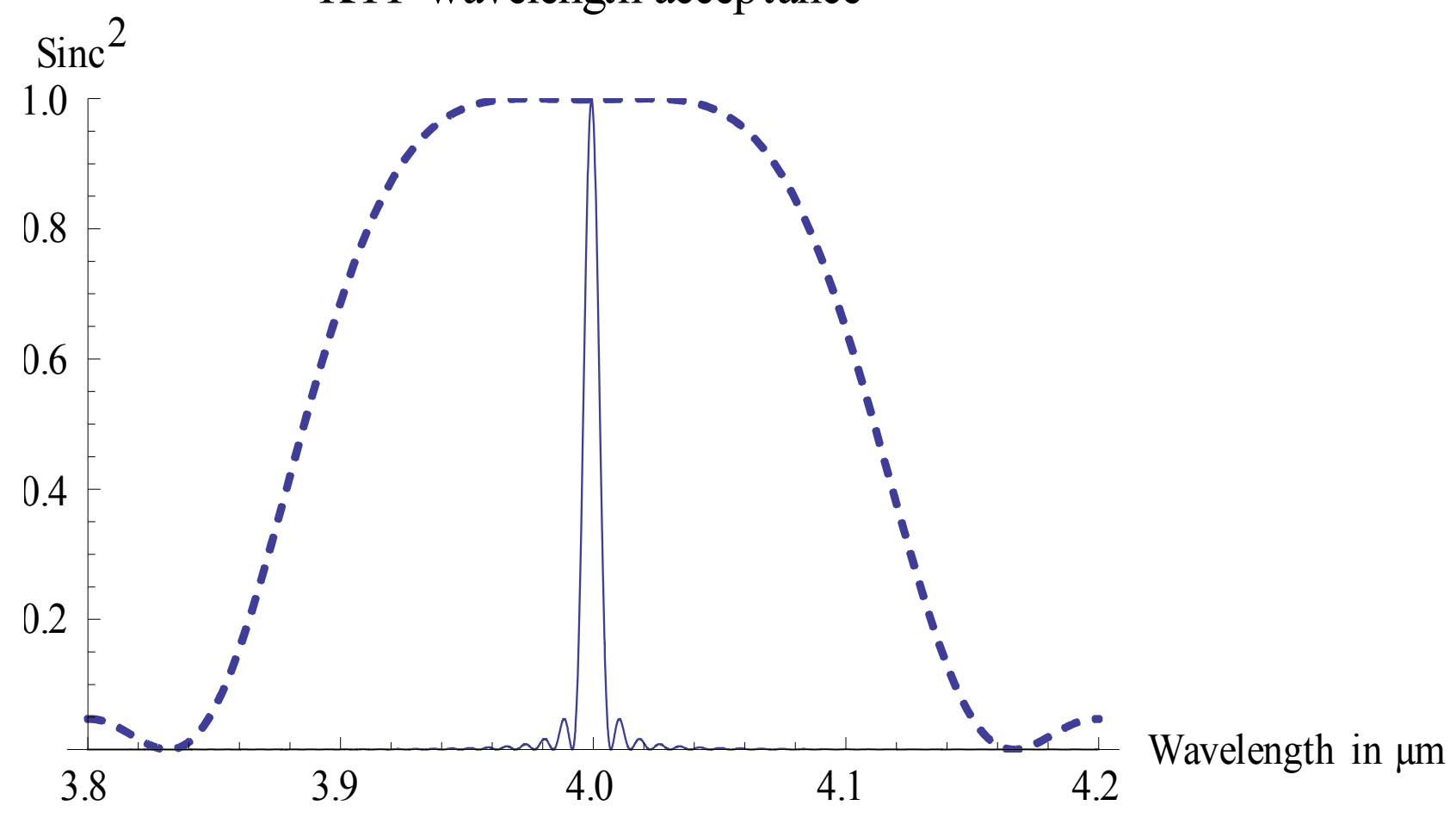

Figure 2. Sum frequency generation of $4 \mu \mathrm{m}$ radiation with $532 \mathrm{~nm}$ light (solid line) or $835 \mathrm{~nm}$ light (dashed). Clearly, very large difference in acceptance bandwidth can be obtained by properly choosing the mixing wavelength for upconversion.

Our recent improvements on quantum efficiency [5] and obtainable resolution [6] give promise of an efficient infrared imaging system based on an image upconversion module. Using the principles described here makes it possible to design the effective bandwidth of the upconversion module.

\section{CONCLUSION}

In this work, we have shown a simple way to analyze and design an infrared image upconversion system to be either fairly broadband, or narrowband. Of course, in practice this mixing wavelength choice is limited somewhat in terms of available laser wavelengths, particularly if high field strengths (quantum efficiencies) are required. The same calculations can be performed for other non-linear crystals suitable for quasi phase matching, since they may provide more suitable wavelength combinations.

We emphasize that the method presented here, does not in itself negatively affect the obtainable quantum efficiencies. If a pulsed laser was used for upconversion, adiabatic phase matching [8] could be used as an alternative method to obtain broadband upconversion. However, since incoherent light is usually only slowly varying in time, this would result in much lower time-averaged quantum efficiencies.

\section{REFERENCES}

[1] J. E. Midwinter, "Image conversion from $1.6 \mu$ to the visible in lithium niobate", Appl. Phys. Lett. 12, 68 (1968). 
[2] J. Warner, "Spatial resolution measurements in up-conversion from $10.6 \mathrm{~m}$ to the visible", Appl. Phys. Lett. 13, 360 (1968).

[3] J. F. Weller, and R. A. Andrews, "Resolution measurements in parametric upconversion of images", Optoelectronics 2, 171 (1970).

[4] R. W. Boyd, and C. H. Townes, "An infrared upconverter for astronomical imaging", Appl. Phys. Lett. 31, 440 (1977).

[5] C. Pedersen, E. Karamehmedović, J. S. Dam, and P. Tidemand-Lichtenberg, "Enhanced 2D-image upconversion using solid-state lasers", Opt. Express 17, 20885-20890 (2009).

[6] J. S. Dam, C. Pedersen, P. Tidemand-Lichtenberg, "High-resolution two-dimensional image upconversion of incoherent light", Opt. Lett. 35, 3796-3798 (2010).

[7] K. Kato and E. Takaoka, "Sellmeier and Thermo-Optic Dispersion Formulas for KTP," Appl. Opt. 41, 5040-5044 (2002).

[8] G. Porat, H. Suchowski, Y. Silberberg, and A. Arie, "Tunable upconverted optical parametric oscillator with intracavity adiabatic sum-frequency generation”, Opt. Lett. 35, 1590-1592, (2010). 